\title{
Supplement: Effects of Seasonal Drawdowns on Fish Assemblages in Sections of an Impounded River-Canal System in Upstate New York
}

Supplementary Table S.1. Approximate physical characteristics of the impoundments of the Barge Canal, New York, where fish communities were surveyed in 2014-2015. Data were obtained from McBride (1987, 1994) and the New York State Canal Corporation.

\begin{tabular}{|c|c|c|c|c|c|}
\hline Impoundment & $\begin{array}{l}\text { Drawdown } \\
\text { amount (m) }\end{array}$ & Area $\left(\mathrm{km}^{2}\right)$ & $\begin{array}{c}\text { Elevation } \\
\text { change at lower } \\
\text { bounding lock } \\
\text { (m) }\end{array}$ & $\begin{array}{c}\text { \% Shipping } \\
\text { channel }\end{array}$ & $\begin{array}{c}\text { River } \\
\text { kilometers at } \\
\text { lower bounding } \\
\text { lock }\end{array}$ \\
\hline Locks 6-7 & 0.5 & 7.7 & 10.1 & 12.1 & 3.5 \\
\hline Locks 7-8 & 0.6 & 4.3 & 8.2 & 24.4 & 21.0 \\
\hline Locks 8-9 & 3.7 & 1.4 & 4.3 & 34.5 & 38.7 \\
\hline Locks 9-10 & 3.9 & 1.8 & 4.6 & 34.3 & 46.8 \\
\hline Locks 10-11 & 5.7 & 1.5 & 4.6 & 26.3 & 56.4 \\
\hline Locks 11-12 & 5.3 & 1.8 & 3.7 & 25.1 & 63.2 \\
\hline Locks 12-13 & 4.3 & 2.5 & 3.4 & 38.2 & 70.0 \\
\hline Locks 13-14 & 3.7 & 1.8 & 2.4 & 43.0 & 85.5 \\
\hline Locks 14-15 & 3.2 & 0.7 & 2.4 & 45.3 & 98.1 \\
\hline Locks $15-16$ & 3.6 & 1.6 & 2.4 & 41.9 & 103.5 \\
\hline Locks 16-17 & 2.0 & 1.0 & 6.2 & 52.9 & 114.3 \\
\hline Locks $17-18$ & 0.5 & 0.6 & 12.3 & 58.6 & 127.1 \\
\hline Locks 18-19 & 2.5 & 1.4 & 6.1 & 54.3 & 133.9 \\
\hline Locks 19-20 & 2.5 & 1.1 & 6.4 & 50.0 & 153.0 \\
\hline Locks 20-Rome & 0.0 & 1.1 & 4.9 & 46.3 & 169.5 \\
\hline
\end{tabular}


Supplementary Table S.2. Catch per unit effort (CPUE; fish/h) and standard errors (SEs) for all species captured from 24 study sites on the Barge Canal, by river section. Underlining denotes species that are not native to the Mohawk River watershed; bold type denotes species for which CPUE was greatest in the seasonally impounded section.

\begin{tabular}{|c|c|c|c|c|c|c|}
\hline \multirow[b]{3}{*}{ Species } & \multicolumn{6}{|c|}{ Section } \\
\hline & \multicolumn{2}{|c|}{ Permanent upper } & \multicolumn{2}{|c|}{ Seasonal } & \multicolumn{2}{|c|}{ Permanent lower } \\
\hline & CPUE & SE & CPUE & SE & CPUE & SE \\
\hline Black Crappie Pomoxis nigromaculatus & 0.3 & 0.01 & 0.0 & 0.00 & 0.0 & 0.00 \\
\hline Bluegill Lepomis macrochirus & 4.3 & 0.07 & 1.2 & 0.04 & 13.7 & 0.37 \\
\hline Bluntnose Minnow Pimephales notatus & 2.0 & 0.05 & 0.4 & 0.01 & 1.7 & 0.07 \\
\hline Brook Silverside Labidesthes sicculus & 0.3 & 0.01 & 0.0 & 0.00 & 0.0 & 0.00 \\
\hline$\overline{\text { Brown Bullhead Ameiurus nebulosus }}$ & 1.3 & 0.05 & 0.9 & 0.03 & 22.6 & 0.41 \\
\hline Brown Trout Salmo trutta & 0.1 & 0.01 & 0.1 & 0.01 & 0.0 & 0.00 \\
\hline$\overline{\text { Chain Pickerel Esox niger }}$ & 2.4 & 0.04 & 0.3 & 0.01 & 0.4 & 0.02 \\
\hline Channel Catfish Ictalurus punctatus & 0.0 & 0.00 & 0.0 & 0.00 & 2.0 & 0.09 \\
\hline Common Carp Cyprinus carpio & 13.9 & 0.18 & 9.6 & 0.11 & 20.0 & 0.27 \\
\hline Emerald Shiner Notropis atherinoides & 3.8 & 0.16 & 0.8 & 0.02 & 12.6 & 0.76 \\
\hline Fallfish Semotilus corporalis & 1.8 & 0.03 & 7.2 & 0.10 & 1.5 & 0.04 \\
\hline Fathead Minnow Pimephales promelas & 0.1 & 0.01 & 0.0 & 0.00 & 0.0 & 0.00 \\
\hline Freshwater Drum Aplodinotus grunniens & 1.1 & 0.02 & 0.7 & 0.02 & 13.5 & 0.35 \\
\hline Gizzard Shad Dorosoma cepedianum & 0.1 & 0.01 & 0.0 & 0.00 & 0.0 & 0.00 \\
\hline$\overline{\text { Golden Shiner Notemigonus crysoleucas }}$ & 0.3 & 0.01 & 0.7 & 0.01 & 9.4 & 0.34 \\
\hline Goldfish Carassius auratus & 0.0 & 0.00 & 0.0 & 0.00 & 1.7 & 0.07 \\
\hline Green Sunfish Lepomis cyanellus & 1.6 & 0.05 & 0.0 & 0.00 & 0.0 & 0.00 \\
\hline Largemouth Bass Micropterus salmoides & 2.4 & 0.04 & 0.1 & 0.01 & 1.5 & 0.05 \\
\hline Logperch Percina caprodes & 4.2 & 0.06 & 7.1 & 0.08 & 10.7 & 0.29 \\
\hline Northern Hog Sucker Hypentelium nigricans & 0.1 & 0.01 & 0.5 & 0.01 & 0.0 & 0.00 \\
\hline Northern Pike Esox lucius & 0.4 & 0.01 & 0.5 & 0.01 & 0.2 & 0.01 \\
\hline$\overline{\text { Pumpkinseed Lepomis gibbosus }}$ & 13.0 & 0.21 & 0.4 & 0.02 & 10.4 & 0.39 \\
\hline Redbreast Sunfish Lepomis auritus & 0.3 & 0.01 & 0.0 & 0.00 & 0.0 & 0.00 \\
\hline Rock Bass Ambloplites rupestris & 17.7 & 0.24 & 0.9 & 0.02 & 9.8 & 0.12 \\
\hline Rosyface Shiner Notropis rubellus & 0.5 & 0.02 & 10.3 & 0.18 & 0.0 & 0.00 \\
\hline Shorthead Redhorse Moxostoma macrolepidotum & 4.2 & 0.07 & 5.6 & 0.12 & 6.1 & 0.12 \\
\hline Smallmouth Bass Micropterus dolomieu & 25.1 & 0.22 & 14.9 & 0.12 & 28.1 & 0.27 \\
\hline Spotfin Shiner Cyprinella spiloptera & 4.4 & 0.08 & 15.6 & 0.15 & 25.2 & 0.40 \\
\hline Spottail Shiner Notropis hudsonius & 11.5 & 0.41 & 0.4 & 0.02 & 1.5 & 0.05 \\
\hline Tessellated Darter Etheostoma olmstedi & 1.0 & 0.03 & 1.1 & 0.02 & 0.2 & 0.01 \\
\hline Tiger Muskellunge E. lucius $\times$ E. masquinongy & 0.1 & 0.01 & 0.0 & 0.00 & 0.0 & 0.00 \\
\hline Walleye Sander vitreus & 11.1 & 0.11 & 2.7 & 0.05 & 8.1 & 0.25 \\
\hline White Bass Morone chrysops & 0.3 & 0.01 & 0.0 & 0.00 & 0.0 & 0.00 \\
\hline White Crappie Pomoxis annularis & 0.8 & 0.04 & 0.0 & 0.00 & 0.0 & 0.00 \\
\hline White Perch Morone americana & 4.3 & 0.08 & 0.0 & 0.00 & 0.6 & 0.02 \\
\hline White Sucker Catostomus commersonii & 8.7 & 0.09 & 6.9 & 0.07 & 8.5 & 0.23 \\
\hline Yellow Bullhead Ameiurus natalis & 0.1 & 0.01 & 0.0 & 0.00 & 0.0 & 0.00 \\
\hline Yellow Perch Perca flavescens & 34.7 & 0.38 & 0.8 & 0.02 & 8.5 & 0.14 \\
\hline
\end{tabular}




\section{SUPPLEMENTAL REFERENCES}

McBride, N. D. 1987. Substrate classification for exposed portions of the partially dewatered lower Mohawk River from Locks 8 to 16. New York State Department of Environmental Conservation, Stamford.

McBride, N. D. 1994. A fisheries management plan for the lower Mohawk River. New York State Department of Environmental Conservation, Albany. 